\title{
A GENERALIZATION OF THE RING OF TRIANGULAR MATRICES
}

\author{
STEPHEN U. CHASE
}

\section{Introduction}

Let $R$ be a ring with unit, and $e$ be an idempotent in $R$ such that $(1-e) R e=0$. In this note we shall explore the relationships between homological properties of $R$ and those of its subring $e R e$.

Examples of such rings are abundant, the most common being perhaps the ring $R$ of all two-by-two upper triangular matrices over a field, where-

$$
e=\left(\begin{array}{ll}
1 & 0 \\
0 & 0
\end{array}\right)
$$

In fact, it is easy to see that every ring of the type described above is in some sense a ring of upper triangular matrices, an observation which justifies the title of this paper.

We exhibit two applications of our results. First, we construct an example of a left semi-hereditary ring which is not right semi-hereditary, thus providing a negative answer to a question of Cartan and Eilenberg ([2], p. 15). Our second application is related to the work of Jans and Nakayama ([5]) and Nakano ([6]) on a class of semi-primary rings which is a special case of the type of ring considered here (recall that a ring $R$ is semi-primary if its Jacobson radical $N$ is nilpotent and $R / N$ satisfies minimum condition on left ideals). Our systematic treatment of the more general situation described above enables us to easily derive- and in some cases strengthen-several of the results of these authors.

Throughout this note every ring will be assumed to have a unit which acts as the identity on all modules. A ring $R$ will be called semi-simple if it has global dimension zero, or, equivalently, it satisfies minimum condition on left ideals and has trivial Jacobson radical ([2], p. 11). $R$ will be called regular 
if it has weak global dimension zero ([2], p. 122).

\section{The Triangular Matrix Construction}

Let $e$ be an idempotent in a ring $R$ with the property that $e^{\prime} R e=0$, where $e^{\prime}=1-e$. Set $S=e R e, S^{\prime}=e^{\prime} R e^{\prime}$.

Proposition 2.1. The mapping $f: R \rightarrow S$ defined by $f(a)=$ eae is a ring epimorphism.

Proof. $f$ is clearly onto, and preserves addition. If $a, b \in R$, then $f(a b)=$ $e(a b) e=(e a)\left(e+e^{\prime}\right)(b e)=(e a e)(e b e)=f(a) f(b)$, since $e$ is an idempotent, $e+$ $e^{\prime}=1$, and $e^{\prime} b e=0$.

Now let $A$ be a left $S$-module. The epimorphism $f: R \rightarrow S$ defines in the usual way a left $R$-module structure on $A$; we shall denote this $R$-module, constructed from $A$, by $T(A)$. On the other hand, if $B$ is a left $R$-module, then $U(B)=e B$ is, in the usual way, a left $S$-module. We have thus defined functors $T: \mathscr{C}(S) \rightarrow \mathscr{C}(R), U: \mathscr{C}(R) \rightarrow \mathscr{C}(S)$, where $\mathscr{C}(R)$ and $\mathscr{C}(S)$ are the categories of all left modules over $R$ and $S$, respectively.

THEOREM 2.1. In the situation described above, the functors $T_{*}$ and $U$ are additive and exact, and $U T$ is naturally equivalent to the identity functor on $\mathscr{C}(S)$. Furthermore, if $A$ is a left $S$-module, then $h d_{R} T(A)=h d_{S} A$.

Proof. That $T$ and $U$ are additive is clear from the definitions. It is also easy to see that $T$ is exact and $U T$ is naturally equivalent to the identity functor on $\mathscr{C}(S)$. Let $A \stackrel{\alpha}{\longrightarrow} B \stackrel{\beta}{\longrightarrow} C$ be an exact sequence of left $R$-modules, and set $\alpha^{*}=U(\alpha), \beta^{*}=U(\beta)$. We have the sequence $U(A) \stackrel{\alpha^{*}}{\longrightarrow} U(B) \stackrel{\beta^{*}}{\rightarrow} U(C)$, which we must show is exact. Recall that $U(A)=e A$ and $\alpha^{*}(e a)=e \alpha(a)$ for $a \in A ; U(B), U(C)$ and $\beta^{*}$ are similarly defined. If $e a \in U(A)$, then $\beta^{*} \alpha^{*}(e a)=$ $\beta^{*}\{e \alpha(a)\}=e(\beta \alpha)(a)=0$, since $\beta \alpha=0$. On the other hand, suppose that $\beta^{*}(e b)=0$, where $e b \in U(B), b \in B$. Then $0=\beta^{*}(e b)=e \beta(b)=\beta(e b)$, and so $e b=\alpha(a)$ for some $a \in A$, since $\operatorname{ker}(\beta)=\alpha(A)$. Then $e a \in U(A)$, and $\alpha^{\psi}(e a)=$ $e \alpha(a)=e(e b)=e b$. Hence the sequence $U(A) \stackrel{\alpha^{*}}{\rightarrow} U(B) \stackrel{\beta^{*}}{\rightarrow} U(C)$ is exact, com. pleting the proof that $U$ is an exact functor.

We claim now that, if $A$ is a left $S$-module, then $T^{\prime}(A)$ is $R$-projective if and only if $A$ is $S$-projective. For observe that $R=R e \oplus R e^{\prime}=e \operatorname{Re} \oplus \operatorname{Re}^{\prime} \approx T(S) \oplus$ $R e^{\prime}$ as a left $R$-module, since $e^{\prime} R e=0$; i.e., $S$, viewed as a left $R$-module, is a 
direct summand of $R$. It then follows by a direct sum argument that $T(A)$ is $R$-projective if $A$ is $S$-projective, since it is easily seen that $T$ preserves arbitrary direct sums. Conversely, suppose that $T(A)$ is $R$-projective. Consider a diagram of the following type-

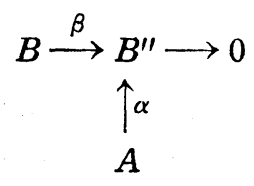

where $B$ and $B^{\prime \prime}$ are left $S$-modules, and the row is exact. Applying $T$, we get the following diagram of $R$-modules-

$$
\begin{gathered}
T(B) \underset{T(\beta)}{\longrightarrow} T\left(B^{\prime \prime}\right) \longrightarrow 0 \\
\uparrow \quad T(\alpha) \\
T(A)
\end{gathered}
$$

The row is exact, since $\mathrm{T}$ is an exact functor. Since $T(A)$ is $R$-projective, there exists an $R$-homomorphism $\theta: T(A) \rightarrow T(B)$ which, when inserted in the above diagram, renders it commutative. Then, applying $U$ and using the fact that $U T$ is naturally equivalent to the identity functor on $\mathscr{C}(S)$, we get a commutative diagram-

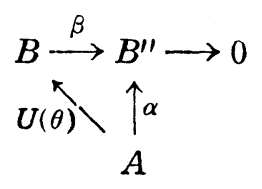

from which it follows that $A$ is $S$-projective.

Now let $A$ be any left $S$-module, and $0 \rightarrow K \rightarrow P \rightarrow A \rightarrow 0$ be exact, where $P$ is a projective left $S$-module. We then get the exact sequence $0 \rightarrow T(K) \rightarrow$ $T(P) \rightarrow T(A) \rightarrow 0$ of left $R$-modules. We have from our previous remarks that $T(P)$ is $R$-projective, and one sequence splits if and only if the other splits. It then follows by an easy induction argument that $h d_{R} T(A)=h d_{s} A$, completing the proof of the theorem.

There are analogous statements describing the relationship between $R$ and $S^{\prime}$. We state these without proof.

Proposition 2.2. The mapping $f^{\prime}: R \rightarrow S^{\prime}$ defined by $f(a)=e^{\prime} a e^{\prime}$ is a ring epimorphism. 
Theorem 2.2. Let $\mathscr{C}^{\prime}(R)$ and $\mathscr{C}^{\prime}(S)$ be the categories of all right modules over $R$ and $S^{\prime}$, respectively. Define functors $T^{\prime}: \mathscr{C}^{\prime}\left(S^{\prime}\right) \rightarrow \mathscr{C}^{\prime}(R)$ and $U^{\prime}$ : $\mathscr{C}^{\prime}(R) \rightarrow \mathscr{C}^{\prime}\left(S^{\prime}\right)$ in entirely similar fashion to the definitions of $T$ and $U$ on $\mathscr{C}(S)$ and $\mathscr{C}(R)$. Then $T^{\prime}$ and $U^{\prime}$ are additive and exact, and $U^{\prime} T^{\prime}$ is naturally equivalent to the identity functor on $\mathscr{C}^{\prime}\left(S^{\prime}\right)$. Furthermore, if $A$ is a right $S^{\prime}$. module, then $h d_{R} T^{\prime}(A)=h d_{s^{\prime}} A$.

Now, changing our point of view, let $S$ and $S^{\prime}$ be arbitrary rings with unit, and $M$ be an $\left(S, S^{\prime}\right)$-bimodule. Let $R$ be the ring of all two-by-two matrices of the form-

$$
\left(\begin{array}{ll}
a & u \\
0 & b
\end{array}\right) \quad a \in S, b \in S^{\prime}, u \in M
$$

where addition is defined to be component-wise, and multiplication is defined by the rule-

$$
\left(\begin{array}{ll}
a & u \\
0 & b
\end{array}\right)\left(\begin{array}{cc}
a^{\prime} & u^{\prime} \\
0 & b^{\prime}
\end{array}\right)=\left(\begin{array}{cc}
a a^{\prime} & a u^{\prime}+u b^{\prime} \\
0 & b b^{\prime}
\end{array}\right) .
$$

It is easy to verify that $R$ is an associative ring with unit. Set-

$$
e=\left(\begin{array}{ll}
1 & 0 \\
0 & 0
\end{array}\right) \quad e^{\prime}=1-e=\left(\begin{array}{ll}
0 & 0 \\
0 & 1
\end{array}\right)
$$

$e$ and $e^{\prime}$ are idempotents in $R$, and $e^{\prime} R e=0 . \quad$ Furthermore, $S \approx e R e, S^{\prime} \approx e^{\prime} R e^{\prime}$, and $M \approx e R e^{\prime}$ as an $\left(S, S^{\prime}\right)$-bimodule. We shall write $R=\mathscr{T}\left(S, S^{\prime}, M\right) . R, S$, and $S^{\prime}$ satisfy the conditions of Theorems 2.1 and 2.2. Finally, we have the following result.

THEOREM 2.3. Let $R$ be a ring, and $e$ be an idempotent in $R$ such that $e^{\prime} R e=0$, where $e^{\prime}=1-e$. Let $S=e R e, S^{\prime}=e^{\prime} e^{\prime}$, and $M=e R e^{\prime}$. Then the mapping $g: R \rightarrow \mathscr{T}\left(S, S^{\prime}, M\right)$ defined by-

$$
g(a)=\left(\begin{array}{cc}
e a e & e a e^{\prime} \\
0 & e^{\prime} a e^{\prime}
\end{array}\right) \quad a \in R
$$

is an isomorphism.

Proof. Routine computation. 


\section{A Left Semi-Hereditary Ring which is Not Right Semi-hereditary}

We are now ready to construct the previously mentioned example of a left semi-hereditary ring which is not right semi-hereditary. Let $S^{\prime}$ be any commutative ring which is regular but not semi-simple (e.g., the direct product of an infinite number of copies of a field). Let $I$ be an ideal in $S^{\prime}$ which is not a direct summand of $S^{\prime}$; such an ideal exists, since $S^{\prime}$ is not semi-simple. Set $S=S^{\prime} / I . \quad S$ is a regular ring, since it is a residue class ring of the regular ring $S^{\prime}$. We may view $S$ as an $\left(S, S^{\prime}\right)$-bimodule. Observe that $S$ is not projective as a right $S^{\prime}$-module, since $I$ is not a direct summand of $S^{\prime}$. Set $R=$ $\mathscr{T}\left(S, S^{\prime}, \mathrm{S}\right)$.

Proposition 3.1. $R$ is left semi-hereditary but not right semi-hereditary.

Proof. Define $\mathscr{C}(R), \mathscr{C}(S), T, U$, etc., as in Theorems 2.1 and 2.2. Let $J^{\prime}$ be the set of all elements of $R$ of the form-

$$
\left(\begin{array}{ll}
0 & u \\
0 & 0
\end{array}\right) \quad u \in S .
$$

It is easy to see that $J^{\prime}$ is a right ideal in $R$, and the mapping $\varphi: T^{\prime}(S) \rightarrow J^{\prime}$ defined by-

$$
\varphi(u)=\left(\begin{array}{ll}
0 & u \\
0 & 0
\end{array}\right) \quad u \in S
$$

is an $R$-isomorphism. Here we are viewing $S$ as an element of $\mathscr{C}^{\prime}\left(S^{\prime}\right)$; i.e., as a right $S^{\prime}$-module. Since $S$ is finitely generated (even cyclic) over $S^{\prime}$, it follows from the definition of $T^{\prime}$ that $T^{\prime}(S)$ is finitely $R$-generated, and so $J^{\prime}$ is a finitely generated right ideal in $R$. But, by Theorem 2.2, we have that $h d_{R} J^{\prime}=h d_{R} T^{\prime}(S)=h d_{S^{\prime}} S>0$. It then follows that $R$ is not right semi-hereditary.

We now show that $R$ is left semi-hereditary. Let $J=\left(\alpha_{1}, \ldots, \alpha_{n}\right)$ be a finitely generated left ideal in $R$, where-

$$
\alpha_{i}=\left(\begin{array}{cc}
a_{i} & u_{i} \\
0 & b_{i}
\end{array}\right) \quad a_{i} \in S, u_{i} \in S, b_{i} \in S^{\prime}
$$

Since $S^{\prime}$ is regular, it follows that $S^{\prime} b_{1}+\ldots+S^{\prime} b_{n}=S^{\prime} e$ for some idempotent $e$ in $S^{\prime}$. Write $\lambda_{1} b_{1}+\ldots+\lambda_{n} b_{n}=e$, where $\lambda_{i} \in S^{\prime}$; then- 


$$
\left(\begin{array}{cc}
0 & 0 \\
0 & \lambda_{i} b_{i}
\end{array}\right)=\left(\begin{array}{cc}
0 & 0 \\
0 & \lambda_{i}
\end{array}\right)\left(\begin{array}{cc}
a_{i} & u_{i} \\
0 & b_{i}
\end{array}\right)=\left(\begin{array}{cc}
0 & 0 \\
0 & \lambda_{i}
\end{array}\right) \alpha_{i} \in J
$$

Hence, setting -

$$
\varepsilon=\left(\begin{array}{ll}
0 & 0 \\
0 & e
\end{array}\right)
$$

we get that $\varepsilon$ is in $J$, and so $R \varepsilon \subseteq J$. Observe that $R \varepsilon$ consists of all elements of $R$ of the form-

$$
\left(\begin{array}{ll}
0 & u e \\
0 & b e
\end{array}\right) \quad u \in S . b \in S^{\prime}
$$

Since $b_{i}=b_{i} e$ for all $i=1, \ldots, n$, we then get that-

$$
\left(\begin{array}{cc}
a_{i} & u_{i}(1-e) \\
0 & 0
\end{array}\right)=\left(\begin{array}{cc}
a_{i} & u_{i} \\
0 & b_{i}
\end{array}\right)-\left(\begin{array}{cc}
0 & u_{i} e \\
0 & b_{i} e
\end{array}\right) \in J
$$

Let $L$ be the left $S$-submodule of $S \oplus S$ generated by the elements $\left(a_{i}, u_{i}(1-e)\right)$, $i=1, \ldots, n$. Define a mapping $h: T(L) \oplus R \varepsilon \rightarrow R$ by-

$$
h\{(u, v)+\alpha \varepsilon\}=\left(\begin{array}{ll}
u & v \\
0 & 0
\end{array}\right)+\alpha \varepsilon=\left(\begin{array}{cc}
u & v+x e \\
0 & b e
\end{array}\right)
$$

where-

$$
\alpha=\left(\begin{array}{ll}
a & x \\
0 & b
\end{array}\right) \quad a \in S, x \in S, b \in S^{\prime} .
$$

It is easily verified that $h$ is a left $R$-module homomorphism, and Image $(h) \subseteq$ J. Also-

$$
h\left(a_{i}, u_{i}(1-e)\right)=\left(\begin{array}{cc}
a_{i} & u_{i}(1-e) \\
0 & 0
\end{array}\right)
$$

and so, since $R \varepsilon \subseteq$ Image $(h)$, it follows from $(*)$ and $(* *)$ above that Image $(h)=J$.

Suppose now that $h\{(u, v)+\alpha \varepsilon\}=0$, where $(u, v) \in L$ and

$$
\alpha=\left(\begin{array}{ll}
a & x \\
0 & b
\end{array}\right) .
$$

It follows from the definition of $L$ that $v=v(1-e)$, and so we have- 


$$
h\{(u, v)+\alpha \varepsilon\}=\left(\begin{array}{ll}
u & v \\
0 & 0
\end{array}\right)+\alpha \varepsilon=\left(\begin{array}{cc}
u & v(1-e)+x e \\
0 & b e
\end{array}\right)=0 .
$$

Then $u=b e=v(1-e)+x e=0$, from which it follows immediately that $v=$ $v(1-e)=0$ and $x e=0$, since $e$ is an idempotent in $S^{\prime}$. Therefore $(u, v)=0$, and-

$$
\alpha \varepsilon=\left(\begin{array}{ll}
a & x \\
0 & b
\end{array}\right)\left(\begin{array}{ll}
0 & 0 \\
0 & e
\end{array}\right)=\left(\begin{array}{ll}
0 & x e \\
0 & b e
\end{array}\right)=0
$$

Hence $(u, v)+\alpha \varepsilon=0$. It then follows that $h$ is an isomorphism; i.e., $J \approx T(L) \oplus$ $R \varepsilon$ as a left $R$-module. Now, $L$ is a finitely generated submodule of the free left $S$-module $S \oplus S$; thus, since $S$ is regular and therefore left semi-hereditary, we have that $L$ is $S$-projective. Then, since $R \varepsilon$ is a direct summand of $R(\varepsilon$ being an idempotent), it follows from Theorem 2.1 that $h d_{R} J=h d_{R} T(L)=$ $h d_{S} L=0$. Therefore every finitely generated left ideal in $R$ is projective, and so $R$ is left semi-hereditary. This completes the proof of the proposition.

\section{Applications to Semi-Primary Rings}

In general it seems to be difficult to express the global dimension of $R=$ $\mathscr{T}\left(R^{\prime}, S, A\right)$ in terms of the homological invariants of $R^{\prime}, S$, and $A$. However. it is easy to obtain complete information for the special case in which $R$ is semi-primary and $S$ is semi-simple; this information will play a key role in our later results on semi-primary rings.

Lemma 4.1. Let $R^{\prime}$ be a semi-primary ring with radical $N^{\prime}$, S be a semisimple ring, and $A$ be an $\left(R^{\prime}, S\right)$-bimodule. Set $R=\mathscr{T}\left(R^{\prime}, S, A\right)$. Then $R$ is a semi-primary ring with radical $N$ consisting of all elements of $R$ of the form-

$$
\left(\begin{array}{ll}
a^{\prime} & x \\
0 & 0
\end{array}\right) \quad a^{\prime} \in N^{\prime}, x \in A .
$$

Furthermore, gl. $\operatorname{dim} . R=\max \left\{\operatorname{gl} \cdot \operatorname{dim} . R^{\prime}, 1+h d_{R^{\prime}} A\right\}$.

Proof. Let $f^{\prime}: R^{\prime} \rightarrow R^{\prime} / N^{\prime}$ be the canonical homomorphism, and define a mapping $f: R \rightarrow\left(R^{\prime} / N^{\prime}\right) \oplus S$ by-

$$
f\left\{\left(\begin{array}{ll}
a^{\prime} & x \\
0 & c
\end{array}\right)\right\}=\left(f^{\prime}\left(a^{\prime}\right), c\right) \quad a^{\prime} \in R^{\prime}, x \in A, c \in S .
$$


It is easily verified that $f$ is an epimorphism with kernel $N$. Another routine computation establishes the fact that, if $\left(N^{\prime}\right)^{r}=0$, then $N^{r+1}=0$. Since $\left(R^{\prime} / N^{\prime}\right)$ D $S$ is semi-simple, it then follows that $R$ is a semi-primary ring with radical $N$.

Now let $\mathscr{C}\left(R^{\prime}\right)$ and $\mathscr{C}(R)$ be the categories of left $R^{\prime}$-modules and left $R$-modules, respectively, and define the functors $T: \mathscr{C}\left(R^{\prime}\right) \rightarrow \mathscr{C}(R)$ and $U$ : $\mathscr{C}(R) \rightarrow \mathscr{C}\left(R^{\prime}\right)$ as in Theorem 2.1. Define a mapping $g: N \rightarrow T\left(N^{\prime}\right) \oplus T(A)$ by-

$$
g\left\{\left(\begin{array}{cc}
a^{\prime} & x \\
0 & 0
\end{array}\right)\right\}=\left(a^{\prime}, x\right) \quad a^{\prime} \in R^{\prime}, x \in A .
$$

It is easy to see that $g$ is a left $R$-module isomorphism. Hence, using Theorem 2.1, we get that $h d_{R} N=\max \left\{h d_{R} T\left(N^{\prime}\right), h d_{R} T(A)\right\}=\max \left\{h d_{R^{\prime}} N^{\prime}, h d_{R^{\prime}} A\right\} . \quad$ But, since $R$ and $R^{\prime}$ are semi-primary rings, we have that $\mathrm{gl} \cdot \operatorname{dim} . R=1+h d_{R} N$ and gl. $\operatorname{dim} . R^{\prime}=1+h d_{R^{\prime}} N^{\prime}$ (see [1]). We then get that gl. $\operatorname{dim} . R=1+h d_{R} N=1+$ $\max \left\{h d_{R^{\prime}} N^{\prime}, h d_{R^{\prime}} A\right\}=\max \left\{1+h d_{R^{\prime}} N^{\prime}, 1+h d_{R^{\prime}} A\right\}=\max \left\{\operatorname{gl} . \operatorname{dim} . R^{\prime}, 1+h d_{R^{\prime}} A\right\}$, completing the proof of the lemma.

Remark. It will be noted that in the statement and proof of the above lemma we have made no distinction between the left and right global dimensions of $R$. This is permissible in view of the fact that, since $R$ is a semi-primary ring, both the left and right global dimensions of $R$ are equal to the weak global dimension of $R$, and are hence equal to each other (see [1]).

Leмma 4.2. Let $R$ be a semi-primary ring with radical $N$, and $e_{1}, \ldots, e_{r}$ be a complete set of mutually orthogonal primitive idempotents of $R$. Suppose that there exists $s<r$ such that $e_{i} N=0$ for $i>s$ but $e_{j} N \neq 0$ for $j \leq s$. Set $e=$ $e_{s+1}+\cdots+e_{r}, e^{\prime}=1-e, R^{\prime}=e^{\prime} R e^{\prime}, S=e R e$, and $A=e^{\prime} R e$. Then $e R e^{\prime}=0$, and $R \approx \mathscr{T}\left(R^{\prime}, S, A\right)$. Furthermore, $S$ is semi-simple.

Proof. Clearly $e N e \subseteq e N=0$, and so $S=e R e$ is semi-simple. If $j \leq s$ and $i>s$, then $e_{i} N=0, e_{j} N \neq 0$, and so $e_{i} R \neq e_{j} R$ as right $R$-modules. Since $e_{i} R \approx$ $\operatorname{Hom}_{R}\left(R e_{i}, R\right)$ and $e_{j} R \approx \operatorname{Hom}_{R}\left(R e_{j}, R\right)$, it then follows that $R e_{i} \approx R e_{j}$, and thus $R e_{i} / N e_{i} \neq R e_{j} / N e_{j}$. We then obtain that $e_{i} R e_{j} \subseteq N$, since $R / N$ is semisimple. Hence $e R e^{\prime} \subseteq N$, and so $e R e^{\prime} \subseteq e N=0$. We then get from Theorem 2.3 that $R \approx \mathscr{T}\left(R^{\prime}, S, A\right)$, completing the proof of the lemma. 
Definition 4.1. Let $R$ be a semi-primary ring with radical $N . \quad R$ will be called triangular if any complete set $e_{1}, \ldots, e_{r}$ of mutually orthogonal primitive idempotents of $R$ can be indexed so that $e_{i} N e_{j}=0$ whenever $i \geq j$.

Our principal results concerning triangular rings, which parallel the work of Jans and Nakayama ([5]), are summarized in the theorems which follow.

THEOREM 4.1. Let $R$ be a semi-primary ring with radical $N$. Then the following statements are equivalent-

(a) $R$ is triangular.

(b) There exists a complete set $e_{1}, \ldots, e_{n}$ of mutually orthogonal primitive idempotents of $R$ such that $e_{i} N e_{j}=0$ if $i \geq j$.

(c) $\operatorname{gl} \operatorname{dim} .(R / I)<\infty$ for any two-sided ideal $I$ in $R$.

(d) $\operatorname{gl} . \operatorname{dim} .\left(R / N^{2}\right)<\infty$.

If any (and hence all) of these conditions hold, then $\mathrm{gl} . \operatorname{dim} . R<r$, where $r$ is the number of isomorphism classes of simple left $R$-modules.

Proof. (a) $\Rightarrow(\mathrm{b})$ : Obvious.

(b) $\Rightarrow$ (c): Let $R$ satisfy (b). It is then clear that (b) also holds for any epimorphic image of $R$; hence to establish (c) we need only show that $R$ itself has finite global dimension. We shall show that $\operatorname{gl} \operatorname{dim} . R<r$. If $r=1$, it follows easily from (b) that $N=0$, in which case $R$ is semi-simple and so gl. $\operatorname{dim} . R=0$. Proceed by induction on $r$; assume the statement true for $r^{\prime}<r$. By hypothesis, there exists a complete set $e_{1}, \ldots, e_{n}$ of mutually orthogonal primitive idempotents of $R$ such that $e_{i} N e_{j}=0$ if $i \geq j$. Then clearly $e_{n} N=0$. We may assume that, for some integer $k<n, e_{k+1} N=e_{k+2} N=\cdots=e_{n} N=0$, but $e_{i} N \neq 0$ for $i \leq k$. Let $e=e_{k+1}+\cdots+e_{n}$ and $e^{\prime}=1-e$. It then follows from Lemma 4.2 that $e R e^{\prime}=0, S=e R e$ is semi-simple, and $R \approx \mathscr{T}\left(R^{\prime}, S, A\right)$, where $R^{\prime}=e^{\prime} R e^{\prime}$ and $A=e^{\prime} R e$. Observe now that $R^{\prime}$ also satisfies (b) and has fewer than $r$ simple components; hence, by the induction assumption, $R^{\prime}$ is a semi-primary ring and $\mathrm{gl}$. $\operatorname{dim} . R^{\prime}<r-1$. That gl. $\operatorname{dim} . R<r$ then follows immediately from Lemma 4.1.

(c) $\Longrightarrow(d)$ : Obvious.

(d) $\Longrightarrow$ (a): Assume that gl. $\operatorname{dim} .\left(R / N^{2}\right)<\infty$. We first prove that $R$ is triangular on the hypothesis that $N^{2}=0$, Let $e_{1}, \ldots, e_{n}$ be any complete set of mutually orthogonal primitive idempotents of $R$, and set $A_{i}=R e_{i} / N e_{i} . \quad A_{i}$ 
is a simple left R-module. $\quad N e_{i}$ is the kernel of the obvious epimorphism of $R e_{i}$ onto $A_{i}$, and is a semi-simple left $R$-module, since $N^{2}=0$. If $e_{i} N e_{j} \neq 0$, then $A_{i}$ is a direct summand of $N e_{j}$, and so $h d_{R}\left(A_{i}\right)<h d_{R}\left(A_{j}\right)$, since both numbers must be finite. We may assume that the $\left\{e_{i}\right\}$ are indexed so that $h d_{R}\left(A_{i}\right) \geq h d_{R}\left(A_{j}\right)$ if $i \geq j . \quad$ It is then clear that $e_{i} N e_{j}=0$ if $i \geq j$, and hence $R$ is triangular in this case. This part of the proof is essentially the same as in [5].

Suppose now that $N^{2}$ is not necessarily zero. We have from the above paragraph that any complete set $e_{1}, \ldots, e_{n}$ of mutually orthogonal primitive idempotents of $R$ may be indexed so that $e_{i} N e_{j} \subseteq N^{2}$ if $i \geq j$. Assume that $e_{i} N e_{j} \subseteq N^{s}$ whenever $i \geq j$, where $s$ is an integer greater than one. Then $e_{i} N e_{j}=$ $e_{i} N^{s} e_{j}$, and we have-

$$
e_{i} N e_{j}=e_{i} N^{s} e_{j}=\left(e_{i} N^{s-1}\right)\left(\sum_{k=1}^{n} e_{k}\right)\left(N e_{j}\right)=\sum_{k=1}^{n}\left(e_{i} N^{s-1} e_{k}\right)\left(e_{k} N e_{j}\right) .
$$

But if $i \geq k$, then $e_{i} N^{s-1} e_{k} \subseteq N^{s}$. On the other hand, if $k \geq i$, then also $k \geq j$, and then $e_{k} N e_{j} \subseteq N^{2}$. It then follows that $e_{i} N e_{j} \subseteq N^{s+1}$. Hence, by induction, we see that, if $i \geq j$, then $e_{l} N e_{j}$ is contained in every power of $\rightarrow N$, and hence $e_{i} N e_{j}=0$, since $N$ is nilpotent. Therefore $R$ is triangular.

The final contention of the theorem follows immediately from inspection of the above arguments. Thus the proof of the theorem is complete.

Remarks. 1. Let $R$ satisfy the conditions of Theorem 4.1. By a somewhat more careful analysis of the situation described in that theorem, utilizing the basic properties of the triangular matrix construction, it is possible to derive the inequality $\mathrm{gl} . \operatorname{dim} . R \leq \mathrm{gl} . \operatorname{dim} .\left(R / N^{2}\right)<r$, thus improving the estimate of the theorem on the global dimension of $R$. Furthermore, if $\operatorname{gl} \operatorname{dim} .\left(R / N^{2}\right)=m$, then $N^{m+1}=0$ ([3], p. 55). These results were proved by Eilenberg, Nagao, and Nakayama ([4]) for residue class rings of hereditary semi-primary rings.

2. Theorem 4.1 was essentially proved, using somewhat different methods, by Jans and Nakayama ([5]) for rings which, in addition to being semi-primary, satisfy a sort of "splitting" condition tatamount to separability of the residue class ring modulo the radical. Their proofs utilized the above-mentioned results of Eilenberg, Nagao, and Nakayama concerning residue class rings of hereditary semi-primary rings, and were based upon their very interesting observation 
that a triangular ring satisfying the above-described splitting condition is a residue class ring, in a particularly nice way, of a unique hereditary semi-primary ring. The existence of this hereditary "covering" ring is, of course, the most important result of the theory; however, it seems to be the only result for which the extra splitting hypothesis is really necessary.

Next we discuss semi-primary rings with the property that every principal right ideal is projective. Our observations will culminate in the theorem that such rings are triangular, a result which was essentially proved by Nakano ([6]). First, a couple of almost obvious lemmas.

Leмma 4.3. Let $R$ be any ring, and $x \in R$. Then the following conditions are equivalent -

(a) The right ideal $x R$ is projective.

(b) The right annihilator of $x$ is a direct summand of $R$.

(c) There exists an idempotent $e$ in $R$ such that $x e=x$, and if $x a=0$ then $e a=0$.

Proof. The lemma may be established by routine computations, which we omit.

Lemma 4.4. Let $R$ be a ring, and suppose that every principal right ideal in $R$ is projective. If $e$ is an idempotent in $R$, then every principal right ideal in $S=e R e$ is projective.

Proof. We have from our hypotheses and Lemma 4.3 that the right annihilator of every element of $R$ is a direct summand of $R$. It is then a straightforward matter to verify that the same condition holds in $S$. Hence, by Lemma 4.3 , every principal right ideal in $S$ is projective. This completes the proof.

THEOREM 4.2. Let $R$ be a semi-primary ring with the property that every principal right ideal in $R$ is projective. Then $R$ is triangular.

Proof. Let $N$ be the radical of $R$, and $r$ be the number of isomorphism classes of simple left $R$-modules. If $r=1$, then $R / N$ is isomorphic as a right $R$-module to a direct sum of simple right ideals in $R$, each of which is necessarily principal; hence, by our hypothesis, $R / N$ is a projective right $R$-module. 
Thus $N$, viewed as a right ideal in $R$, is a direct summand of $R$, which is impossible unless $N=0$. Therefore $N=0$, in which case $R$ is a simple ring and the theorem is trivially true.

Proceed by induction on $r$; assume the theorem true for $r^{\prime}<r$. If $R$ is semi-simple we are done; otherwise $N \neq 0$ and there exists an element $x \in R$ such that $x N=0$. By hypothesis $x R$ is projective, and so, by Lemma 4.3 , there exists an idempotent $e_{0} \in R$ such that $x e_{0}=x$, and if $x a=0$ then $e_{0} a=0$. Then $x N=0$, and so $e_{0} N=0$. We may write $e_{0}=\bar{e}+e_{1}$, where $\bar{e}$ and $e_{1}$ are orthogonal idempotents and $\bar{e}$ is primitive. Then $\bar{e}=\bar{e} e_{0}$, and so $\bar{e} N=\bar{e} e_{0} N=0$.

Since $R$ is semi-primary, there exists a complete set $e_{1}, \ldots, e_{n}$ of mutually orthogonal primitive idempotents of $R$ such that $e_{n}=\vec{e}$. Since $e_{n} N=\bar{e} N=0$, we may assume that, for some integer $k<n, e_{k+1} N=e_{k+2} N=\cdots=e_{n} N=0$, but $e_{i} N \neq 0$ for $i \leq k$. Let $e=e_{k+1}+\cdots+e_{n}, e^{\prime}=1-e$; we then get from Lemma 4.2 that $S=e R e$ is semi-simple and $R \approx \mathscr{T}\left(R^{\prime}, S, A\right)$, where $R^{\prime}=e^{\prime} R e^{\prime}$ and $A=e^{\prime} R e$. Observe that $R^{\prime}$ has less than $r$ isomorphism classes of simple left modules. By Lemma 4.4, every principal right ideal in $R^{\prime}$ is projective; hence, by the induction assumption, $R^{\prime}$ is a triangular semi-primary ring. It then follows immediately that $R$ itself is triangular, completing the proof of the theorem.

Nakano ([6]) has proved a result which is essentially equivalent to Theorem 4.2, although he replaced the hypothesis that $R$ be semi-primary by the following assumption: $R=I_{1} \oplus \cdots \oplus I_{n}$, where $I_{k}$ is a left ideal in $R$ and $\operatorname{Hom}_{R}\left(I_{k}, I_{k}\right)$ is a sfield. However, it is easy to see that such a ring is actually semi-primary; hence Nakano's result is contained in (and is, in fact, equivalent to) Theorem 4.2. Nakano proved also a sort of converse of Theorem 4.2, which we shall not consider here.

\section{REFERENCES}

[1] M. Auslander, On the Dimension of Modules and Algebras, III: Global Dimension, Nagoya Math. J. vol., 9 (1955), pp. 67-77.

[2] H. Cartan and S. Eilenberg, Homological Algebra, Princeton University Press, 1956.

[3] S. Chase, Module-Theoretic Characterizations of Rings, Ph.D. Thesis, University of Chicago, 1960.

[4] S. Eilenberg, H. Nagao, and T. Nakayama, On the Dimension of Modules and Algebras, IV: Dimension of Residue Rings of Hereditary Rings, Nagoya Math. J. vol., 10 (1956), pp. 87-95. 
[5] J. P. Jans and T. Nakayama, On the Dimension of Modules and Algebras, VII: Algebras with Finite-Dimensional Residue Algebras, Nagoya Math. J. vol., 11 (1957), pp. 67-76.

[6] T. Nakano, A Nearly Semi-Simple Ring, Commentatorium Mathematicorum Universitatis Sancti Pauli vol., 7 (1959), pp. 27-33.

University of Chicago and

Princeton University 